\title{
Determinants of Incident Vulvovaginal Candidiasis in Human Immunodeficiency Virus-Positive Women
}

\author{
Emma Shifrin, ${ }^{1}$ Debra Matityahu, ${ }^{1}$ Joseph Feldman, ${ }^{2}$ and \\ Howard Minkoff ${ }^{1 *}$ \\ ${ }^{1}$ Department of Obstetrics and Gynecology, Maimonides Medical Center, State University of New \\ York Downstate, Brooklyn, NY \\ ${ }^{2}$ Department of Preventive Medicine, State University of New York Downstate, Brooklyn, NY
}

\begin{abstract}
Objective: Mucosal infections including vulvovaginal candidiasis are a common problem for women with human immunodeficiency virus (HIV) infection. Our objective was to determine which factors predict the development of symptomatic disease among HIV-infected women.

Materials and Methods: In a prospective study from 1991 to 1995, 205 HIV-positive women were evaluated every 6 months for occurrences of vulvovaginal candidiasis. Included in the study were all initially asymptomatic women, whether they were fungal-culture-positive or -negative at baseline. Excluded from the study were all women with symptomatic vulvovaginal candidiasis at the initial visit, those who developed trichomonas vaginitis at any visit, and those who used any antifungal agents.

Results: The risk of the development of vulvovaginal candidiasis did not differ between women who were asymptomatically colonized at baseline and those who were fungal-culture-negative. However, the risk of developing vulvovaginal candidiasis was increased 6.8 times for women with CD4 counts less than 200 cells $/ \mathrm{mm}^{3}$ at baseline.

Conclusions: Fungal culture is not predictive of the development of symptomatic vulvovaginal candidiasis. Women infected with HIV who have CD4 counts below 200 cells $/ \mathrm{mm}^{3}$ should be monitored more carefully for vulvovaginal candidiasis. Infect. Dis. Obstet. Gynecol. 8:176-180, 2000. @ 2000 Wiley-Liss, Inc.
\end{abstract}

$\mathrm{V}$ ulvovaginal candidiasis, although not considered a defining illness of acquired immune deficiency syndrome (AIDS), is categorized by the Centers for Disease Control's classification system for human immunodeficiency virus (HIV) infection as a condition whose course or management may be altered by HIV infection. Several studies have reported that vaginal candidiasis among HIV-positive women occurs frequently and is due primarily to Candida albicans and Torulopsis glabrata. ${ }^{1}$ Depressed T-cell function due to drugs or immunological or systemic disease is a well-recognized factor predisposing to either local or diffuse mucosal candidiasis, and cell-mediated immunity depression correlates well with the occurrence of local or systemic candidal infection. ${ }^{2}$ The prevalence of

Grant sponsor: National Institutes of Allergy and Infectious Diseases; grant number: RO1-AI-31834, contract NO1-a195014. Grant sponsor: NICHD; grant number: WESTAT contract NO1-HD-8-2913 and CONRAD Program subproject agreement CS4-94-134.

*Correspondence to: Howard Minkoff, Maimonides Medical Center, 9802 10th Avenue, Brooklyn, NY 11219. E-mail:hminkoff@maimonidesmed.org 
the infection has been related to the immune status of HIV-infected women.

Not all authors have found the same relationship between HIV infection and vulvovaginal candidiasis. ${ }^{3}$ Some have suggested that vaginal candidiasis is not an AIDS-related illness and that the initial studies overestimated its importance among HIVinfected women because of bias in those studies related to the failure to include community controls. However, most authors have found some increase in the prevalence of colonization and disease, particularly among those whose CD4 counts are at the lower extremes. ${ }^{2,4}$

Although several authors have reported the prevalence of vulvovaginal candidiasis, less is known about the incidence and the determinants of symptomatic disease. In an earlier report from the cohort described here, we noted that the incidence of many gynecologic conditions, including Candida, is increased among HIV-infected women. However, the relative contribution of colonization and immune status to incident cases of vulvovaginal candidiasis was not assessed.

Since vulvovaginal candidiasis is a common problem for HIV-positive women, we report here the results of a prospective study of a cohort of HIV-positive women to determine the incidence, and the risk factors associated with the development, of that condition.

\section{SUBJECTS AND METHODS}

From September 1991 through July 1995, a total of 205 HIV-positive women were enrolled in a prospective study of the natural history of HIV disease in women and 197 were available for follow-up. At 6-month intervals, a gynecologic exam was performed, and demographic, behavioral, and clinical variables were assessed. The examinations included fungal cultures. Fungal cultures were collected as follows: a sterile cotton-tipped swab was inserted into the introitus and inoculated onto a plate containing bismuth sulfite-glucose-glycineyeast medium (Remel, Lenexa, KS). The cultures were incubated at $35^{\circ} \mathrm{C}$ and examined daily. Cultures were considered negative when there was no evidence of growth after 7 days of incubation. Yeasts were identified using standard mycologic procedures, including gram staining, germ tube formation, and the API 20C system (Analytab Products, Inc., Plainview, NY) for biochemical reac- tions. Vulvovaginal candidiasis was defined as the presence of $C$. albicans on culture in addition to self-reported symptoms of vaginal burning, itching, or abnormal discharge. Patients were asked about interval visits for candidal symptoms or discharge. Specimens for Trichomonas vaginalis were collected with calcium alginate swabs, placed in tubes of modified Diamond's medium (Remel), and incubated at $35^{\circ} \mathrm{C} \pm 1^{\circ} \mathrm{C}$ for up to 7 days. These cultures were examined daily by wet mount $(110 \times)$ for the presence of typical motile trichomonads, as previously described. ${ }^{5}$ Enumeration of T-cell subsets by flow cytometry using monoclonal antibodies (Becton Dickinson, San Jose, CA) was done at an AC'TU certified laboratory at each study visit. Serologic status for syphilis was assessed using the rapid plasma reagin test (RPR) and confirmed by the Treponema pallidum hemagglutination assay (TPHA).

To focus on incident cases, data were analyzed for: 1) women with a positive culture for Candida but lacking vaginal symptoms at the time of enrollment and 2) women with a negative culture for Candida at the time of enrollment. Symptomatic women who were culture-positive at enrollment were excluded from analysis. Women who had trichomonal vaginitis at any follow-up visit or who reported use of antifungal agents since the prior follow-up were also excluded.

Comparisons between proportions were by Fisher exact test. The probability of developing vulvovaginal candidiasis was estimated by the Kaplan-Meier method. The probability of vulvovaginal candidiasis occurrence adjusted for other variables was determined by Cox proportional hazard model. Results were considered statistically significant at $P<0.05$. In regard to power considerations, overall, there was a $20 \%$ chance of developing vulvovaginal candidiasis during the 2.5-year study period. With the present sample size, there was a $74 \%$ chance of detecting a two-fold or greater difference in the relative risk between the asymptomatic and negative-culture women.

\section{RESULTS}

As noted, all symptomatic women with positive candidal cultures $(n=30)$ and those who developed trichomonas vaginitis during follow up $(n=6)$ or received antifungal medications in the prior 6 months $(n=34)$ were excluded from analysis. One 
TABLE I. Characteristics of Candida-colonized and noncolonized $\mathrm{HIV}$-infected women

\begin{tabular}{|c|c|c|c|}
\hline & $\begin{array}{c}\text { Noncolonized } \\
N=130\end{array}$ & $\begin{array}{c}\text { Colonized } \\
\mathrm{N}=21\end{array}$ & $P$-value \\
\hline Age $(y)$ & $33.2 \pm 7.7$ & $33.1 \pm 10.1$ & 0.94 \\
\hline \multicolumn{4}{|l|}{ Ethnicity (\%) } \\
\hline Hispanic & 22.3 & 23.8 & \\
\hline Black & 70.8 & 71.4 & 0.93 \\
\hline White & 6.9 & 4.8 & \\
\hline \multicolumn{4}{|l|}{ CD4 group (\%) } \\
\hline$<200$ cells $/ \mathrm{mm}^{3}$ & 11.5 & 4.8 & \\
\hline $200-499$ cells $/ \mathrm{mm}^{3}$ & 56.2 & 52.4 & 0.24 \\
\hline $500+$ cells $/ \mathrm{mm}^{3}$ & 32.3 & 42.9 & \\
\hline \multicolumn{4}{|l|}{ Syphilis serology } \\
\hline reactivity (\%) & 15.5 & 33.3 & 0.06 \\
\hline
\end{tabular}

hundred and thirty women who were asymptomatic and were Candida-culture-negative and 21 asymptomatic culture-positive women were included in the analysis. The mean age for both groups was 33 years, with no significant differences in ethnicity or CD4 counts (Table 1).

Of the 130 asymptomatic culture-negative women, 18 developed at least one episode of vulvovaginal candidiasis over the 2.5-year follow-up, and of the 21 asymptomatic culture-positive women, two developed at least one episode of vulvovaginal candidiasis over the 2.5 -year follow-up. There was no difference between the two groups in the frequency of interval visits for Candidarelated symptoms or discharge. The average length of follow up for each group was 1 year. There was not a significant difference in the incidence of vulvovaginal candidiasis between the two groups $(P<$ $0.8)$. After the culture-positive and culturenegative groups were adjusted for age, prior antibiotic use, syphilis reactivity (TPHA), and CD4 group, there was still no significant difference between the incidence of vulvovaginal candidiasis in the two groups $(P<0.45)$.

The two groups were then combined and the relative risks of vulvovaginal candidiasis were then determined in relation to prior positive RPR, prior antibiotic use, and age (Table 2). The history of prior reactive syphilis serology increased the relative risk of developing vulvovaginal candidiasis by 4.1 times $(P=0.01)$. A history of prior antibiotic use since last visit increased the relative risk of vulvovaginal candidiasis 2.2 times $(P=0.12)$. After adjusting for age, prior antibiotic use, and syphilis serology, the relative risk of vulvovaginal candidiasis was 8.2 times greater for women with a CD4
TABLE 2. Risk of incident vulvovaginal candidiasis in 130 women culture-negative at onset and 2 | women culture-positive at onset

\begin{tabular}{lccc}
\hline & $\begin{array}{c}\text { Relative } \\
\text { risk }\end{array}$ & P-value & $\begin{array}{c}95 \% \\
\text { confidence } \\
\text { interval }\end{array}$ \\
Variable & 0.92 & 0.07 & $0.84-1.01$ \\
\hline $\begin{array}{l}\text { Age (y) } \\
\text { History of prior antibiotic use }\end{array}$ & 2.18 & 0.12 & $0.82-5.81$ \\
$\begin{array}{l}\text { Group (asymptomatic vs. } \\
\quad \text { culture-negative) }\end{array}$ & 0.74 & 0.69 & $0.16-3.35$ \\
$\begin{array}{l}\text { Positive RPR } \\
\text { CD4 group } \\
\quad<200 \text { vs. }>500 \text { cells } / \mathrm{mm}^{3}\end{array}$ & 4.18 & 0.01 & $1.45-12.00$ \\
$\quad 200-499$ vs. $>500$ cells $/ \mathrm{mm}^{3}$ & 1.62 & 0.37 & $0.57-4.64$ \\
\hline
\end{tabular}

count less than $200 \mathrm{cells} / \mathrm{mm}^{3}(P=0.02)$ (Figure 1). The risk of vulvovaginal candidiasis among women with a CD4 counts less than 200 cells $/ \mathrm{mm}^{3}$ remained eight times greater in a model stratified by the initial culture status.

\section{DISCUSSION}

We have found that an HIV-infected woman's immune status is a more important determinant of the subsequent occurrence of vulvovaginal candidiasis than is vaginal culture status at baseline. These findings may have important implications for the monitoring and care of HIV-infected women.

A number of prior studies have reported on the prevalence of, and risk factors for, candidiasis in HIV-positive women. Early in the AIDS epidemic, Rhoads et al. studied 29 HIV-positive women at the Walter Reed Army Medical Center and reported that in $24 \%$ chronic candidal vaginitis was the presenting complaint and that it predated the occurrence of oral thrush. ${ }^{6}$ They suggested that in these circumstances vulvovaginal candidiasis might be the only clinical indication of severe underlying immunodeficiency.

Spinillo et al. prospectively studied 84 HIVpositive women and $384 \mathrm{HIV}$-negative controls and found a prevalence of vaginal candidiasis of $61.9 \%$ among the HIV-positive women and $32.3 \%$ in the control group. ${ }^{2}$ They also determined that after treatment of the vaginal infection, the time to recurrence was significantly shorter in HIV-positive patients than in controls and that the time interval correlated with the severity of immunosuppression.

Imam et al. studied $66 \mathrm{HIV}$-positive women over a 3 -year period and demonstrated that Candida often infected the vaginal mucosa before there was 


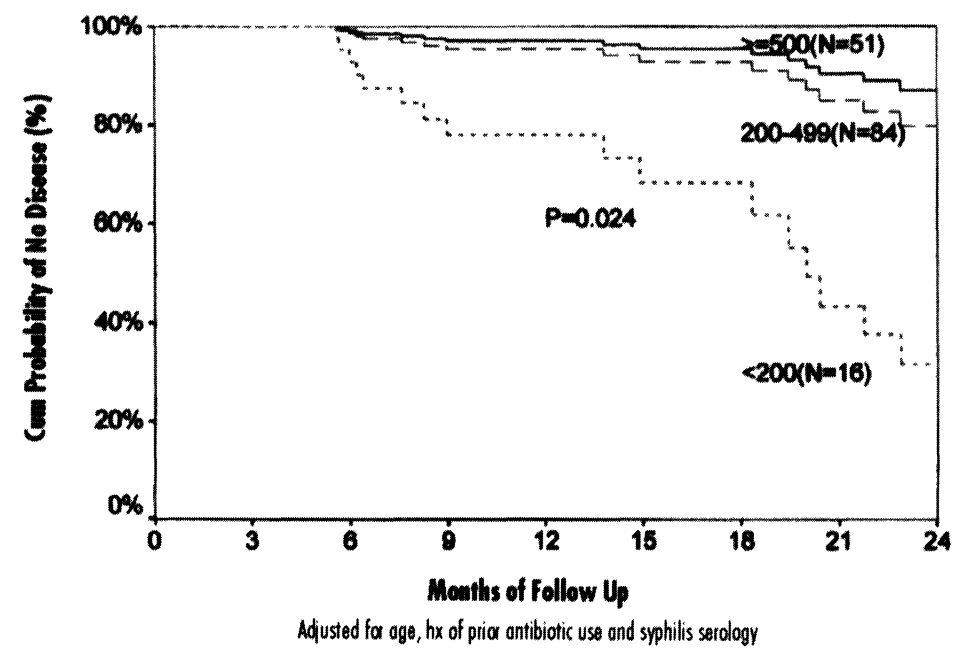

Fig. I. Observed probability of developing vulvovaginal candidiasis by CD4 group $(n=151)$, adjusted for age, history of prior antibiotic use, and syphilis serology.

a significant reduction in CD4 counts. ${ }^{4}$ However, candidal infection of the oropharynx was associated with highly significant reductions in the CD4 count.

More recently, in a previous study of this cohort, it was found that 81 of 223 (36\%) HIV-infected women without AIDS-defining conditions and 72 of 289 (25\%) HIV-uninfected women were culturepositive for vaginal candidiasis. ${ }^{1}$ It was concluded that among HIV-positive women, risks for colonization were increased approximately threefold in women with CD4 counts below 200 cells $/ \mathrm{mm}^{3}$ compared with either immunocompetent HIVinfected or HIV-uninfected women. Another report from this cohort noted that HIV-infected women were also significantly more likely to have symptomatic candidiasis (odds ratio $=1.80 ; 95 \%$ confidence interval, $1.0-3.25 ; P=0.05) .{ }^{7}$

Although many authors have reported on the prevalence of colonization and disease, few have assessed incidence. A previous study that included some of the same women assessed here, did report the incident rate of candidiasis along with many other gynecologic conditions. ${ }^{7}$ However, there was no careful assessment of factors associated with incident vulvovaginal candidiasis.

In the current analysis we have found a $9 \%$ annual incidence of at least one episode of vulvovaginal candidiasis among HIV-positive women. There was no significant difference in the incidence of vulvovaginal candidiasis between HIV-infected women who were asymptomatic and fungal-culture-positive and HIV-infected women who were asymptomatic and fungal-culture-negative, even when adjusting for confounding factors such age, antibiotic use, history of positive RPR, and CD4 count. The association noted with RPR status might reflect small sample size, differences in sexual histories, or a relationship between immune status and RPR status. However, for HIV-positive women with a CD4 count less than 200 cells $/ \mathrm{mm}^{3}$, there was an increase of 8.2 times in the incidence of vulvovaginal candidiasis.

The finding that low CD4 count was more closely associated with the subsequent development of vulvovaginal candidiasis than was colonization with Candida suggests that it would be more appropriate to base monitoring protocols on immune status than on colonization status. Thus, putting an asymptomatic woman with a relatively high CD4 count who is colonized with Candida on prophylactic medications would not seem warranted by these data. Conversely, women with low CD4 counts should be closely monitored for the development of vulvovaginal candidiasis prior to the development of symptoms independent of colonization status.

\section{REFERENCES}

1. Duerr A, Sierra MF, Feldman J, et al. Immune compromise and prevalence of candida vulvovaginitis in human immunodeficiency virus-infected women. Obstet Gynecol 1997;90:252-256.

2. Spinillo A, Michelone G, Cavanna C, et al. Clinical and 
microbiological characteristics of symptomatic vulvovaginal candidiasis in HIV-seropositive women. Genitourin Med 1994;70:268-272.

3. White MH. Is vulvovaginal candidiasis an AIDS-related disease? Clin Infect Dis 1996;22 (suppl 2):S124-127.

4. Imam N, Carpenter CCJ, Mayer KH, et al. Hierarchical pattern of mucosal Candida infections in HIVseropositive women. Am J Med 1990;89:142-146.

5. Chirgwin KD, Feldman J, Augenbraun M, et al. Incidence of venereal warts in human immunodeficiency virus-infected and uninfected women. J Infect Dis 1995; 172:235-238

6. Rhoads JL, Wright DC, Redfield RR, Burke DS. Chronic vaginal candidiasis in women with human immunodeficiency virus infection. JAMA 1987;257:31053107.

7. Minkoff H, Feldman J, Burke R, et al. The prevalence and incidence of gynecologic disease among HIVinfected and uninfected women. Am J Obstet Gynecol 1999;180:824-836. 


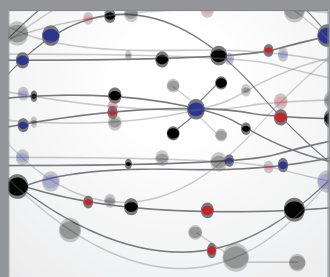

The Scientific World Journal
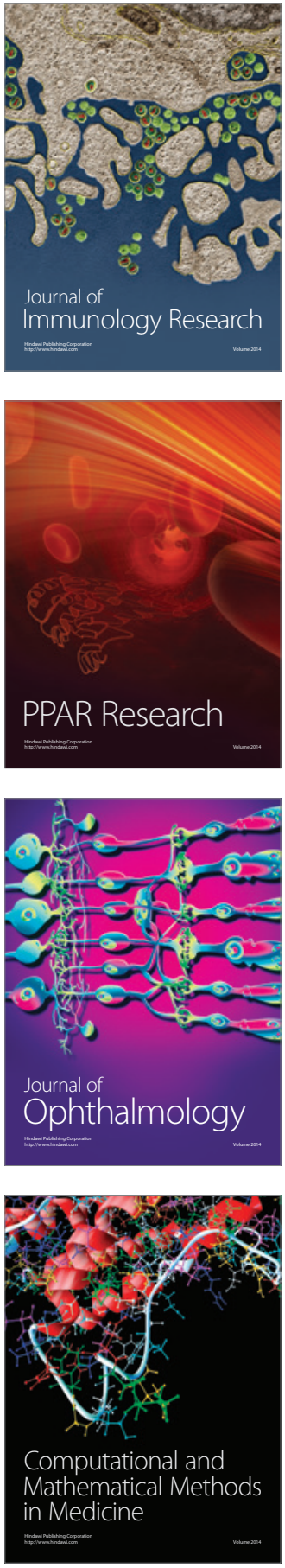

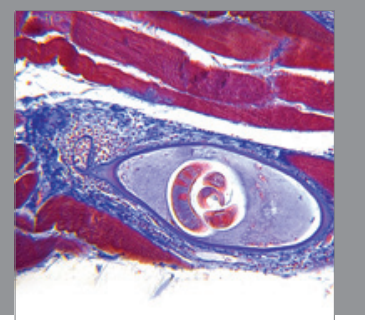

Gastroenterology

Research and Practice
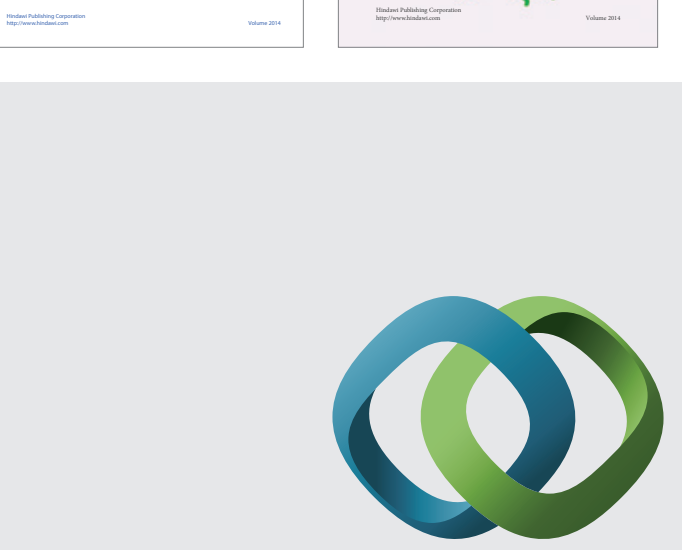

\section{Hindawi}

Submit your manuscripts at

http://www.hindawi.com
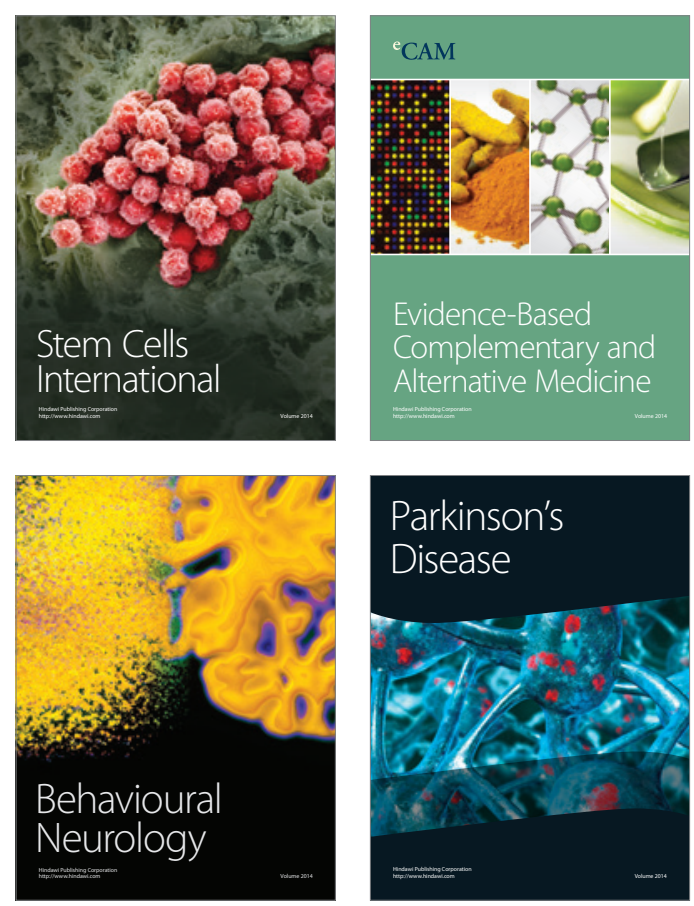

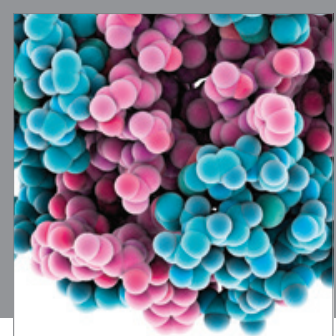

Journal of
Diabetes Research

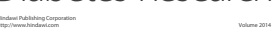

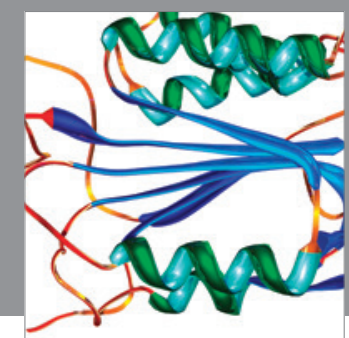

Disease Markers
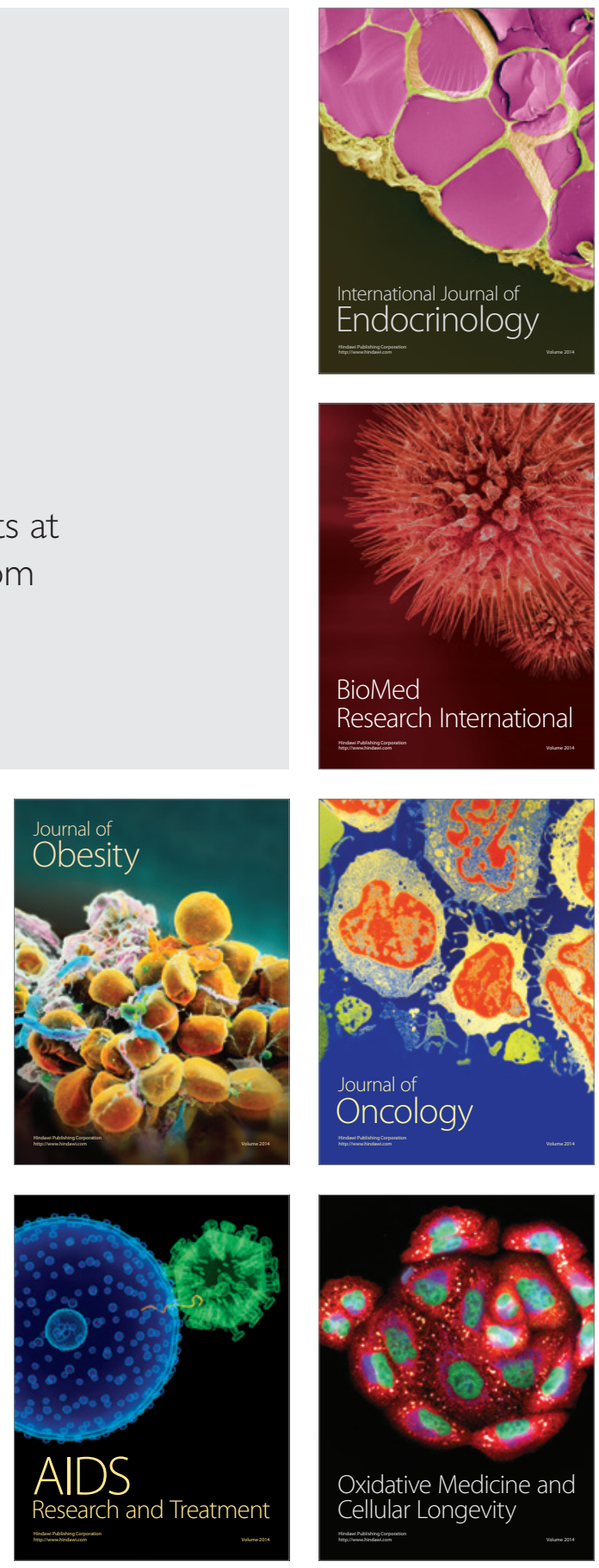